\title{
Tracheostomy in spinal cord injured: frequency and follow up
}

\author{
M Biering-Sørensen MD, F Biering-Sørensen MD PhD \\ Centre for Spinal Cord Injured, Rigshospitalet, National University Hospital, Havnevej \\ 25, DK-3100 Hornbaek, Denmark.
}

\begin{abstract}
Among 600 traumatic spinal cord injured (SCI) patients admitted during a 20 year period, 67 had a tracheostomy performed $(11.2 \%)$. Of these 67 patients 46 had a cervical SCI. A concomitant thoracic trauma had occurred significantly more often in the group with thoracic or lumbar SCI than in those with a cervical SCI. The period from injury to tracheostomy was 0-48 days (median 4.4 days), and from tracheostomy to decannulation 3-167 days (median 31 days). At follow up $20(30 \%)$ had died, primarily because of respiratory problems. Of the remaining 47 patients, $43(91 \%)$ responded to a follow up questionnaire 3.5-21.7 years (median 10.4 years) after the injury. In the follow up 53\% reported never to have had any inconvenience or trouble related to the tracheostomy. The major inconvenience among the others had been of cosmetic origin $(28 \%)$, and 3 had had a surgical revision for this reason. At the time of follow up 9 patients $(21 \%)$ still had certain complaints, primarily described as difficulty in swallowing. Minitracheostomy might in the future reduce the number of these complications.
\end{abstract}

Key words: spinal cord injury; respiratory problems; tracheostomy; frequency; complications; follow up.

\section{Introduction}

Respiratory problems are among the major threats to acute spinal cord injured (SCI) patients, ${ }^{1-6}$ and tracheostomy is regularly required, particularly in tetraplegic patients. ${ }^{1,7}$

The purpose of the present study was to estimate the frequency of tracheostomy in a 20 year series of acute traumatic SCI patients admitted to the Rehabilitation Hospital in Hornbæk, Denmark. A further follow up was carried out to identify possible complications and later treatments because of the tracheostomies.

\section{Methods}

All 600 records on traumatic SCI patients admitted to the Rehabilitation Hospital in Hornbæk, Denmark during the years

Correspondence to: Maja Biering-Sørensen MD, Centre for Spinal Cord Injured, Rigshospitalet, National University hospital, Havnevej 25, DK-3100 Hornbæk, Denmark.
1968-1987 were reviewed. In the study patients with a traumatic spinal cord or cauda equina lesion were included.

For all patients we determined the level of the spinal cord lesion as the most caudally normally functioning spinal cord segment at injury, and the functional level (Frankel class $)^{8}$ at the latest follow up. The records for those who had a tracheostomy performed were further reviewed to determine age at the time of injury; the level of fracture, if any; the cause of injury; and thoracic trauma (including costal fracture(s), pneumo- or haemothorax) at the time of injury. The time from injury to tracheostomy, any complications, and the time from tracheostomy to decannulation were registered. For those who died, the time from injury to death was calculated, and the cause of death was identified in death certificates from the National Board of Health and in available autopsy or discharge reports.

Patients alive at follow up were questioned in accordance with a fixed questionnaire by phone if possible, otherwise by 
mail. They were asked if they found that the tracheostomy had meant any discomfort to them, in particular if any respiratory stenosis or cosmetic problems could possibly be related to the tracheostomy. If later treatments including operations were carried out because of the tracheostomy, these were reviewed and the effect recorded.

Fisher's exact test, chi-square, and MannWhitney rank-sum test have been used in statistical comparisons, and 5\% was chosen as the level of significance.

\section{Results}

In all 67 patients had a tracheostomy performed, ie $11.2 \%(67 / 600)$ of all the traumatic SCI patients (Table I). Fifty-six were men and 11 women. Cervical lesions were significantly ( $p=0.00037)$ more common in the group who had tracheostomies performed compared to the others.

Concomitant thoracic trauma was present in 9 of the cervical SCI, 12 of the thoracic, and all 3 lumbar SCI, ie significantly more thoracic traumas among the lower lesions compared with the cervical SCI ( $p=$ $0.0001)$. In addition those with thoracic trauma were significantly older at the time of injury compared with those without thoracic trauma $(p=0.038)$. The 6 thoracic SCI without concomitant thoracic trauma had neurological levels Th3-Th8, 5 Frankel class $\mathrm{A}$ and one $\mathrm{B}$.

Twelve had no spinal fracture, 36 had a cervical fracture, 15 a thoracic, and 4 a lumbar fracture.

Age at injury is shown in Figure 1. The median age at injury was 34.4 years, the range 5-69 years.

The cause of injury was a traffic accident in 40 patients, in 10 a fall to a level below, in 7 diving into shallow water, in 3 sporting accidents, and in another 3 attempted suicide.

The functional level according to Frankel et $\mathrm{al}^{8}$ at the latest follow up examination can be seen in Table I. For the cervical lesions tracheostomies were significantly more frequent $(p=0.01)$ among those with the

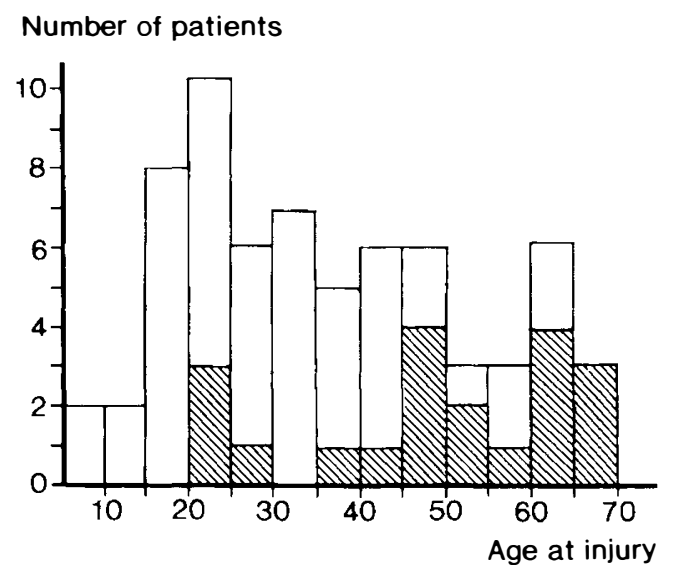

Figure 1 Age at spinal cord injury. The hatched area represent those patients who were dead at the time of follow up.

Table I Neurological level at injury and Frankel scale ${ }^{8}$ at the latest follow up examination for the 67 spinal cord injured (SCI) patients who had a tracheostomy performed, compared to all 600 traumatic SCI patients admitted during the years 1968-87. The number of tracheostomised SCI patients, the number of all SCI patients and the corresponding percentage are shown for each group.

\begin{tabular}{|c|c|c|c|c|c|c|}
\hline $\begin{array}{l}\text { Neurological } \\
\text { level }\end{array}$ & A & B & $\begin{array}{l}\text { e according } \\
\quad \mathrm{C}\end{array}$ & $\begin{array}{l}\text { to Frankel et al } \\
\text { D }\end{array}$ & E & Total \\
\hline & $\%$ & $\%$ & $\%$ & $\%$ & $\%$ & $\%$ \\
\hline Cervical & $16 / 71$ & $8 / 48(17)$ & $7 / 27(26)$ & $14 / 103(14)$ & $1 / 39(3)$ & $46 / 288(16)$ \\
\hline Thoracic & $13 / 117(11)$ & $1 / 29$ & $0 / 16(0)$ & $4 / 27$ & $0 / 11(0)$ & $18 / 200 \quad(9)$ \\
\hline Lumbar* & $0 / 11 \quad(0)$ & $0 / 10 \quad(0)$ & $0 / 15 \quad(0)$ & $2 / 40 \quad(5)$ & $1 / 36(3)$ & $3 / 112$ \\
\hline Total & 29/199 (15) & $9 / 87(10)$ & $7 / 58(12)$ & $20 / 170(12)$ & $2 / 86(2)$ & $67 / 600$ (11) \\
\hline
\end{tabular}

*including 3 sacral lesions Frankel E in the all-SCI group. 
more severe injuries, according to the Frankel classification.

The number of days from injury to tracheostomy was in median 4.4 days (range $0-48)$. Three had their tracheostomies performed on the day of injury, 6 the day after, and 122 days post injury. Two were not tracheostomised before 32 and 48 days after injury; according to the records the final decision to perform tracheostomy was made because of long term respirator treatment. For 2 patients the dates for tracheostomy and decannulation are unknown and in one the date of decannulation is unknown; all 3 patients were tracheostomised abroad (Sweden, Germany and England) and no detailed records from these hospitalisations are available.

The period until decannulation was 31 days in median (range 3-167 days, 25 percentile 19 days, 75 percentile 47 days).

Three patients had each one of the following early complications to the tracheostomy: bleeding from the stoma, pneumothorax, and pneumomediastinum.

Before follow up 20 had died, ie $30 \%$ $(20 / 67)$. The age at injury was significant $(p=0.00009)$ higher among those who died before the follow up (median 49.0 years, range 22-69 years) compared to the others (median 28.7 years, range 5-65 years) (Fig 1). The period from injury to death was in median 7.9 years (range 2.3 months -19.4 years), and the age at death was 56.7 years in median (range 23.1-82.8 years). The information on neurological level at injury, the frequency of thoracic trauma or spinal fractures, the cause of injury, the Frankel class, the periods from injury to tracheostomy and from tracheostomy to decannulation showed no statistically significant differences between those who died and those who were still alive at follow up. The death certificates revealed that 17 had died from cardiorespiratory problems with the following reported diagnoses: pneumonia ( 6 patients); respiratory insufficiency (4 patients - one had chronic bronchitis, another was intubated shortly before death, and a third had cardiac arrhythmia); acute myocardial infarction ( 3 patients - one also had aggressive chronic lymphatic leukaemia); pulmonary oedema ( 2 patients - one had acute tracheobronchitis); incompensated heart (1 patient); and 'heart stop' (one patient). One died because of septicaemia due to a sacral decubitus, but also had at the time of death bilateral bronchopneumonia. Another died with hyperpyrexia of unknown etiology, but had a decubitus, and autopsy revealed pulmonary stasis. The last patient died 2 days following a cerebral haemorrhage.

Follow up answers were obtained from 43 of the 47 surviving patients $(91 \%)$. The 4 for whom no follow up data are available are all living abroad (Australia, England, Greenland, and Syria). The time from injury to follow up was 10.4 years in median (range $3.5-21.7$ years), and the age at follow up was 41.8 years in median (range 19.9-78.9 years). Twenty three, ie $53 \%$, reported that they never had any inconvenience or trouble because of the tracheostomy.

Only one patient, a 38 years old, C7 Frankel class $\mathrm{C}$, stated at the follow up that she had respiratory stenosis problems, but also had bronchitis.

Twelve patients $(28 \%)$ reported that some cosmetic inconvenience had occurred, and in 3 instances with a very deep scar, excision followed by suture was performed 10 months, $3 \frac{1}{2}$ years, and $13 \frac{1}{2}$ years after the tracheostomy. All operations had a good result. One more patient had asked for revision of the scar.

Ten patients $(23 \%)$ stated that from time to time they had a tight feelings in relation to the scar, and the majority of those reported a simultaneous difficulty in swallowing. Six of the 10 patients also reported cosmetic inconvenience, and one was among those who had the scar surgically revised. Another of these patients had a nice looking scar, but it was fixed to the larynx. He had prednisolone injected into the scar 16 months after the tracheostomy, resulting in the disappearance of the problem.

Apart from the above, only a few minor problems were reported to appear after tracheostomy.

At the time of the follow up 9 patients $(21 \%)$ still had some problems which they ascribed to the previously performed tracheostomy, including difficulty in swallowing (6 
patients), cosmetic inconvenience (3 patients - one had asked for surgical revision), other troubles (4 patients) and combined problems were reported.

\section{Discussion}

The patients admitted to the Rehabilitation Hospital in Hornbæk are representative of patients with traumatic SCI who survive the acute period after injury, and who do not require respiratory support afterwards; although patients with less severe SCI not requiring specialised treatment were not admitted.'

These admission criteria have to be taken into consideration when the frequency of performed tracheostomies in our patients $(11.2 \%)$ is compared with materials including the acute period. Bellamy et $a l^{1}$ found that $77 \%$ with complete, and $33 \%$ with incomplete quadriplegia had a tracheostomy performed, including the $40 \%$ of the complete and $21 \%$ of the incomplete who died in the first year. It is not possible to calculate the frequency of tracheostomy among the patients who survived the acute period. Cheshire ${ }^{7}$ reported that tracheostomy was required in 6 of 20 patients (ie $30 \%$ ) with tetraplegia, complete and incomplete together. It may not be surprising that we find lower frequencies, ie $16 \%$ for all tetraplegics, and $21 \%$ if we exclude those with Frankel class D-E (Table I). Because of the span of years between the previous studies $^{1,7}$ and ours, a more frequent initial use of ventilation by nasopharyngeal or oral intubation, and the increasing use of periodic CPAP (continuous positive airway pressure) by a mask in recent years ${ }^{10}$ might have reduced the need for some tracheostomies. This might be supported by the more recent study by Reines and Harris ${ }^{5}$ who found a frequency of tracheostomies on $9 \%$, even when they included those patients who died.

The period from injury to tracheostomy is clearly longer in our series compared to that of Bellamy et al. ${ }^{1}$ The reason is probably that nasopharyngeal or oral intubation have been used for ventilation for a longer period in our population before tracheostomy was performed. ${ }^{5}$

The 3 early complications in relation to the tracheostomies in this study agree with those previously reported ${ }^{11}$ as the most frequent: pneumothorax, pneumomediastinum and stomal haemorrhage.

Considering the late complications, we found no serious stenosis problems which could have been expected. ${ }^{12}$ One reason for this might be that those who had developed stenosis were those who died, in particular when considering that the overwhelming cause of death in the follow up period was respiratory complications. Although this is in agreement with previously published studies on the mortality in SCI patients, and in tetraplegics in particular, ${ }^{13-17}$ the very marked dominance in our study is noteworthy.

Cosmetic inconvenience was reported to have been prevalent for $28 \%$ in the follow up period, although only 3 patients $(7 \%)$ at the time of follow up still considered this to be a problem. But 3 patients also had a surgical revision performed in the meantime, and others learned to live with their scars, for instance by using high-necked blouses or shirts.

Another persistent complaint was the feeling of tightness at the location of the scar and swallowing difficulties, and this was still a problem for 6 patients $(14 \%)$ at the time of follow up. Whether these swallowing problems have anything to do with the previously performed tracheostomies is uncertain, and the complaint was not so serious that lateral cervical spine $\mathrm{x}$-rays or other specific investigations required to be carried out.

Although the latter reported problems do not appear to be very serious, they must be considered, and possibly the more widespread use of minitracheostomy ${ }^{18}$ in the early respiratory management of the patients would reduce the number of these complaints, even although it is not possible from the available information in the present study to ascertain whether this procedure would have been effective in reducing the frequency of complications. 


\section{References}

1 Bellamy R, Pitts FW, Stauffer ES (1973) Respiratory complications in traumatic quadriplegia. J Neurosurg 39: 596-600.

2 Carter RE (1987) Respiratory aspects of spinal cord management. Paraplegia 25: 262-6.

3 Fishburn MJ, Marino RJ, Ditunno JF (1990) Atelectasis and pneumonia in acute spinal cord injury. Arch Phys Med Rehabil 71: 197-200.

4 Mansel JK, Noeman JR (1990) Respiratory complications and management of spinal cord injuries. Chest 97: $1446-52$.

5 Reines HD, Harris RC (1987) Pulmonary complications of acute spinal cord injuries. Neurosurgery 21: 193-6.

6 Silver JR, Gibbon NOK (1968) Prognosis in tetraplegia. Br Med J 4: 79-83.

7 Cheshire DJE (1964) Respiratory management in acute traumatic tetraplegia. Paraplegia 1: 252-61.

8 Frankel HL, Hancock DO, Hyslop G, Melzak J, Michaelis LS, Ungar GH et al (1969) The value of postural reduction in the initial management of closed injuries in the spine with paraplegia and tetraplegia. Paraplegia 7: 179-92.

9 Biering-Sørensen F, Pedersen V, Clausen S (1990) Epidemiology of spinal cord lesions in Denmark. Paraplegia 28: 105-18.

10 Andersen JB, Olesen KP, Eikard B, Jansen E, Quist J (1980) Periodic continous positive airway pressure, CPAP, by mask in the treatment of atelectasis. European J Resp Dis 64: 20-5.

11 Piotrowski JJ, Moore EE (1988) Emergency department tracheostomy. Emer Med Clin North Am 6: 737-44.

12 Hsu S, Dreisbach JN, Charlifue SW, English GM (1987) Glottic and tracheal stenosis in spinal cord injured patients. Paraplegia 25: 136-48.

13 DeVivo MJ, Kartus PL, Stover SL, Rutt RD, Fine PR (1989) Cause of death for patients with spinal cord injuries. Arch Intern Med 149: 1761-6.

14 Geisler WO, Jousse AT, Wynne-Jones M, Breithaupt D (1983) Survival in traumatic spinal cord injury. Paraplegia 21: 364-73.

15 Kraus JF, Franti CE, Borhani NO, Riggins RS (1979) Survival with an acute spinal-cord injury. J Chron Dis 32: 269-83.

16 Nakajima A, Honda S, Yoshimura S, Ono Y, Kawamura J, Moriai N (1989) The disease pattern and causes of death of spinal cord injured patients in Japan. Paraplegia 27: 163-71.

17 Nyquist RH, Bors E (1967) Mortality and survival in traumatic myelopathy during nineteen years, from 1946 to 1965 . Paraplegia 5: 22-48.

18 Gupta A, McClelland MR, Evans A, El Masri WS (1989) Minitracheostomy in the early management of patients with spinal injuries. Paraplegia 27: 269-77. 